\title{
Retinal Nerve Fiber Layer Alterations After Photocoagulation: Prospective Spectral-Domain OCT Study
}

\author{
Sitkı Eren, Taylan Ozturk, Aylin Yaman, Hakan Oner and A. Osman Saatci*
}

Department of Ophthalmology, Dokuz Eylul University School of Medicine, Izmir, Turkey

\begin{abstract}
Purpose: To evaluate the effect of panretinal photocoagulation (PRP) on retinal nerve fiber layer thickness (RNFLT) in treatment-naive patients with proliferative diabetic retinopathy (PDR).

Methods: Fifty eight previously untreated eyes of 30 patients with PDR who underwent PRP treatment were enrolled prospectively. All patients had at least six months of follow-up. Detailed ophthalmologic examinations including macular thickness and RNFLT assessments with spectral-domain type optic coherence tomography were performed at baseline as well as the third and sixth posttreatment months. Initial RNFLT and macular thickness of laser administered patients were compared with two separate control groups that were consisted of either nondiabetic patients or diabetics without PDR.

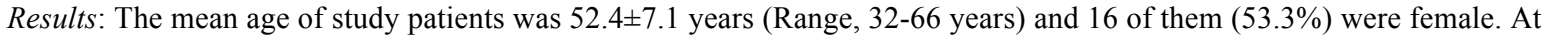
the sixth post-PRP month, visual stabilization or improvement was achieved in 54 eyes $(93.1 \%)$. No significant difference was demonstrated in initial RNFLT measurements between the study patients and two control groups $(\mathrm{p}=0.478)$. Mean RNFLT was measured as $108.5 \pm 17.5 \mu \mathrm{m}, 115.8 \pm 17.6 \mu \mathrm{m}$, and $103.0 \pm 16.4 \mu \mathrm{m}$ at baseline, third and sixth months of the follow-up, respectively. Although RNFLT increase noted at the third post-laser month was statistically significant compared to its baseline values $(\mathrm{p}<0.001)$, there was a significant reduction in RNFLT at the sixth post-laser month compared to its baseline values $(\mathrm{p}<0.001)$.

Conclusion: RNFLT increase in the third month of follow-up may be related to ensuing axonal edema. Significant RNLFT decrease at the sixth month of follow-up may be attributed to axonal loss secondary to the laser treatment.
\end{abstract}

Keywords: Diabetic retinopathy, panretinal photocoagulation, retinal nerve fiber layer.

\section{INTRODUCTION}

Diabetic retinopathy (DR) is one of the most important facet of diabetes, and a major cause of visual loss and blindness in individuals between 20 and 65 years of age [1]. According to Diabetic Retinopathy Study Group (DRS), severe visual loss (acuity poorer than 5/200) may occur in $37 \%$ of untreated eyes having high-risk characteristics of proliferative diabetic retinopathy (PDR) within six years $[2$, 3]. Panretinal laser photocoagulation (PRP) is still the gold standard for the treatment of PDR. Melanosomes located within the retinal pigment epithelium (RPE) absorbe the laser energy that causes a thermal injury and coagulates the adjacent photoreceptors and RPE cells. As photoreceptors are metabolically the most active cells in retina with high oxygen consumption, photocoagulation lowers the metabolic load and reduces the ischemia and ischemia-driven angiogenic substances. Increased oxygenation and metabolic transport from the choroidea to the inner retinal layers are also achieved by creating photoreceptor-free glial window defects with laser burns [4].

Although conventional photocoagulation primarily affects RPE and photoreceptors, laser burns may damage the

*Address correspondence to this author at the Mustafa Kemal Pasa Bulvari, No:73, A blok, Daire: 9, Narlidere, Izmir, 35320, Turkey;

Tel: +905055251600; Fax: +902324123099;

E-mail: osman.saatci@deu.edu.tr inner retina and even nerve fiber layer as well [5]. Mild retinal edema may occur soon after the PRP as laser treatment triggers retinal inflammation due to increased vascular permeability. However in the long-term, reduction in retinal nerve fiber layer thickness (RNFLT) due to the damaged retinal cells including photoreceptors and ganglion cells is likely to happen [6-10]. Spectral-domain (SD) type optic coherence tomography (OCT) provides distinct advantages in signal-to-noise ratio permitting faster signal acquisition, optimal repeatability and reproducibility in measuring RNFLT. Previous studies showed excellent reproducibility of RNFLT measurements with SD-OCT in both normal and glaucomatous eyes [11-13]. In our prospective study, RNFLT alterations relevant to PRP were evaluated via SD-OCT in patients with treatment naive proliferative DR.

\section{MATERIALS AND METHODOLOGY}

Fiftyeight treatment naive eyes of 30 patients with PDR who underwent PRP at our retina clinic between September 2011 and June 2012 (Group I) were prospectively enrolled. The mean scores of initial RNFLT and central macular thickness (CMT) were compared with two age and sexmatched control groups, one of which was consisted of 60 eyes of 30 nondiabetic subjects (Group II), and the other, 60 eyes of 30 diabetic patients without DR (Group III). Patients with concomitant ocular pathologies such as glaucoma, corneal opacity, uveitis, degenerative myopia, age-related 
macular degeneration, hypertensive retinopathy, and retinal dystrophies; cases who underwent cataract surgery within 6 months, as well as patients requiring combined treatment with intravitreal injections were excluded. Informed consent conforming to the tenets of the Declaration of Helsinki were obtained from each study participant, under protocols approved by local ethics committee (Approval number and date: 2011/23-03; 07.07.2011). All patients had at least six months of follow up after the completion of PRP. Detailed ophthalmologic examinations including best-corrected visual acuity (BCVA), slit-lamp biomicroscopy, ocular tonometry, dilated fundoscopy, CMT and RNFLT assessments with SDOCT were performed prior to the enrollment, as well as at the third and sixth months after the PRP. Retinal nerve fiber layer (RNFL) circular scans were performed using the Spectralis SD-OCT system (Heidelberg Engineering, Heidelberg, Germany) after the pupillary dilation. For the initial measurement of RNFLT, the OCT scanning circle was manually positioned at the center of the optic disc while eye tracking was activated. The first acquired RNFL scan was set as a baseline for further retest scans.

In 58 eyes of 30 patients with proliferative DR (Group I), PRP was performed using a frequency-doubled Nd:YAG$532 \mathrm{~nm}$ laser (532 nm; VISULAS 532; Zeiss; Carl Zeiss Meditec, Jena, Germany) with the help of widefield Mainster PRP lens (Ocular Instruments, Inc., Bellevue, WA, USA) and Goldmann three-mirror lens (Ocular Instruments, Inc., Bellevue, WA, USA). On average, patients in Group I received a total of $1576.6 \pm 312.0$ laser spots with sufficient energy to cause a medium white burn with one burn width spacing. PRP was completed in 3-4 sittings, with a one-week interval between each sitting, and the patients were reevaluated at the third and sixth months after the completion of PRP. Visual improvement as well as changes in CMT and RNFLT were compared at each posttreatment visit. Main outcome measure of the present study was the change in RNFLT following the PRP. The changes in both CMT and BCVA after laser treatment were the secondary outcomes.

The data was stored on a computerized database and analyzed using SPSS 16.0 for Windows (Statistical Package for Scientific Studies for Windows, SPSS Inc., Chicago, IL, USA). Student's t test, paired t test, Friedman test, One way ANOVA and Pearson chi-square tests were used in the statistical analysis where appropriate and a value of below 0.05 was considered as significant.

\section{RESULTS}

The mean age in Group I was 52.4 \pm 7.1 years (Range, 3266 years). There was 16 females $(53.3 \%)$ and 14 males (46.7\%). Twentynine patients $(96.7 \%)$ had type-2 diabetes, and the mean diabetes duration was $12.6 \pm 6.5$ years (Range, 2-23 years). No statistically significant difference was found among three study subgroups with respect to age and gender $(p=0.998$ and $p=0.732)$. No statistically significant difference was also found in initial RNFLT scores among three study subgroups (One way ANOVA; $\mathrm{p}=0.478$ ). Mean CMT score was $260.4 \pm 32.8 \mu \mathrm{m}$ (Range, 217-326 $\mu \mathrm{m}$ ), 235.9 $\pm 24.6 \mu \mathrm{m}$

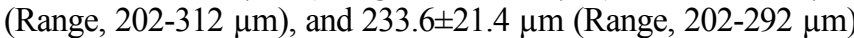
in Group I, II, and III, respectively. While there was no statistically significant difference in initial CMT scores between the control groups (Student's $\mathrm{t}$ test; $\mathrm{p}=0.580$ ), patients in Group I had higher CMT values (One way ANOVA; $\mathrm{p}<0.001)$. Demographics as well as initial RNFLT and CMT scores of 3 study subgroups were summarized in Table 1.

When evaluating quadrant by quadrant, the nasal RNFLT increased significantly from $81.3 \pm 16.5 \mu \mathrm{m}$ prior to PRP to 93.2 $\pm 20.6 \mu \mathrm{m}$ at the third post-laser month (Paired t test; $\mathrm{p}=0.004$ ) in Group I. In the inferior and superior quadrants, the RNFLT increased from $128.3 \pm 23.0 \mu \mathrm{m}$ and $127.5 \pm 19.2 \mu \mathrm{m}$ at baseline to $136.0 \pm 22.8 \mu \mathrm{m}$ and $131.1 \pm 19.5 \mu \mathrm{m}$, respectively at the third month after the PRP (Paired t test; $\mathrm{p}<0.001$ and $\mathrm{p}=0.006$ ). However, no statistically significant increase was found in RNFLT scores of temporal quadrant at the third month of laser comparing to its baseline counterpart (Paired t test; $p=0.342$ ). Statistically significant decrease was found in RNFLT at the sixth month of laser comparing to the baseline scores in temporal, nasal, superior, and inferior quadrants (Paired t test; $\mathrm{p}=0.002, \mathrm{p}=0.001 ; \mathrm{p}=0.005$, and $\mathrm{p}<0.001$, respectively). Fluctuations in RNFLT with respect of retinal quadrants were shown in Table 2 and Fig. (1).

In Group I, initial RNFLT was $108.5 \pm 17.5 \mu \mathrm{m}$, whereas it was $115.8 \pm 17.6 \mu \mathrm{m}$ and $103.0 \pm 16.4 \mu \mathrm{m}$ at the third and sixth months after PRP treatment. However significant decrease was found in mean RNFLT at the sixth month of the follow-up when compared with the initial scores (Paired $t$ test; $\mathrm{p}<0.001), 7.3 \mu \mathrm{m}$ of mean RNFLT increase was

Table 1. Demographics and ophthalmologic findings.

\begin{tabular}{|c|c|c|c|c|}
\hline & Group I & Group II & Group III & p Value \\
\hline Age (years) & $52.4 \pm 7.1$ & $52.3 \pm 8.0$ & $52.4 \pm 6.9$ & 0.998 \\
\hline $\begin{array}{l}\text { Gender (n; \%) } \\
\quad \text { Female } \\
\text { Male }\end{array}$ & $\begin{array}{l}14 ; 46.7 \\
16 ; 53.3\end{array}$ & $\begin{array}{l}13 ; 43.3 \\
17 ; 56.7\end{array}$ & $\begin{array}{l}16 ; 53.3 \\
14 ; 46.7\end{array}$ & 0.732 \\
\hline BCVA (logMAR) & $0.30 \pm 0.14$ & $0.26 \pm 0.22$ & $0.28 \pm 0.22$ & 0.463 \\
\hline IOP (mmHg) & $14.8 \pm 2.2$ & $15.0 \pm 2.1$ & $14.6 \pm 2.2$ & 0.566 \\
\hline Mean initial CMT $(\mu \mathrm{m})$ & $\begin{array}{l}260.4 \pm 32.8 \\
(217-326)\end{array}$ & $\begin{array}{l}235.9 \pm 24.6 \\
(202-312)\end{array}$ & $\begin{array}{l}233.6 \pm 21.4 \\
(202-292)\end{array}$ & $<0.001$ \\
\hline Mean initial RNFLT $(\mu \mathrm{m})$ & $\begin{array}{c}108.5 \pm 17.5 \\
(81-160)\end{array}$ & $\begin{array}{c}105.7 \pm 12.6 \\
(85-144)\end{array}$ & $\begin{array}{c}105.8 \pm 12.5 \\
(82-147)\end{array}$ & 0.478 \\
\hline
\end{tabular}


Table 2. Changes in BCVA, mean CMT, and mean RNFLT scores within the follow-up period in Group I.

\begin{tabular}{|c|c|c|c|c|}
\hline & Baseline & 3rd Month & 6th Month & p Values \\
\hline BCVA $(\log M A R)$ & $0.30 \pm 0.14$ & $0.29 \pm 0.13$ & $0.28 \pm 0.12$ & $\begin{array}{l}0.262^{\dagger} \\
0.080^{*} \\
0.218^{*}\end{array}$ \\
\hline Mean CMT $(\mu \mathrm{m})$ & $260.4 \pm 32.8$ & $254.6 \pm 27.1$ & $252.2 \pm 23.9$ & $\begin{array}{c}<0.001^{\dagger} \\
<0.001^{*} \\
0.063^{\ddagger}\end{array}$ \\
\hline Mean total RNFLT $(\mu \mathrm{m})$ & $108.5 \pm 17.5$ & $115.8 \pm 17.6$ & $103.0 \pm 16.5$ & $\begin{array}{l}<0.001^{\dagger} \\
<0.001^{*} \\
<0.001^{*}\end{array}$ \\
\hline Mean temporal RNFLT $(\mu \mathrm{m})$ & $96.3 \pm 36.1$ & $91.4 \pm 19.4$ & $78.6 \pm 16.7$ & $\begin{array}{c}0.342^{\dagger} \\
0.002^{*} \\
<0.001^{*}\end{array}$ \\
\hline Mean nasal RNFLT $(\mu \mathrm{m})$ & $81.3 \pm 16.5$ & $93.2 \pm 20.6$ & $74.5 \pm 17.8$ & $\begin{array}{c}0.004^{\dagger} \\
0.001^{*} \\
<0.001^{*}\end{array}$ \\
\hline Mean inferior RNFLT $(\mu \mathrm{m})$ & $128.3 \pm 23.0$ & $136.0 \pm 22.8$ & $122.2 \pm 21.2$ & $\begin{array}{l}<0.001^{\dagger} \\
<0.001^{*} \\
<0.001^{\ddagger}\end{array}$ \\
\hline Mean superior RNFLT $(\mu \mathrm{m})$ & $127.5 \pm 19.2$ & $133.1 \pm 19.5$ & $122.2 \pm 22.5$ & $\begin{array}{c}0.006^{\dagger} \\
0.005^{*} \\
<0.001^{*}\end{array}$ \\
\hline
\end{tabular}

: Comparison between scores measured at baseline and the third month of follow-up.

: Comparison between scores measured at baseline and the sixth month of follow-up.

${ }^{\ddagger}$ : Comparison between scores measured at the third and sixth months of follow-up.

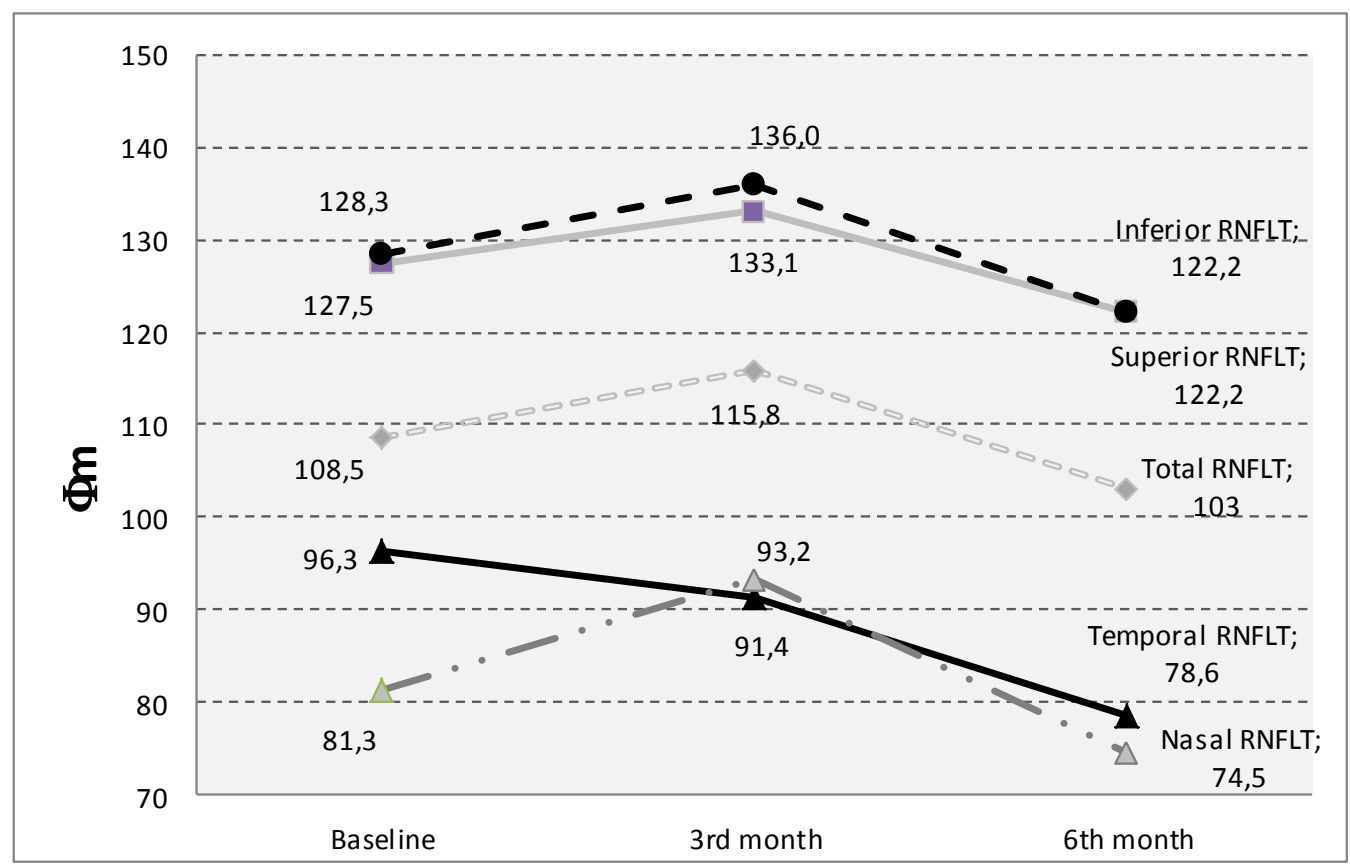

Fig. (1). Alterations in RNFLT with respect of retinal quadrants in patients who underwent PRP treatment.

detected in the first 3 months after PRP treatment (Paired $\mathrm{t}$ test; $\mathrm{p}<0.001)$. Significant decrease in mean RNLFT was also found between the third and sixth month visits (Paired $t$ test; $\mathrm{p}<0.001$ ).
In Group I, initial mean BCVA was $\log \mathrm{MAR} 0.30 \pm 0.14$, whereas BCVA was logMAR $0.29 \pm 0.13$, and $\log$ MAR $0.28 \pm 0.12$ at the third and sixth months of follow-up (Friedman test; $\mathrm{p}<0.001$ ). Visual acuity was decreased in 4 
eyes $(6.9 \%)$, however visual stabilization or improvement were noted in 54 eyes $(93.1 \%)$ six months after the PRP. While initial CMT was 260.4 $\pm 32.8 \mu \mathrm{m}$ (Range, 217-326 $\mu \mathrm{m}$ ), it was 254.6 $\pm 27.7 \mu \mathrm{m}$ (Range, 214-316 $\mu \mathrm{m}$ ) and $252.2 \pm 24.1 \mu \mathrm{m}$ (Range, 212-308 $\mu \mathrm{m}$ ) at the third and sixth months after the PRP (Friedman test; $\mathrm{p}<0.001$ ). Mean CMT at the third and the sixth months following the laser treatment was significantly lower than the initial CMT scores (Paired t test; $\mathrm{p}<0.001$ and $\mathrm{p}<0.001$ ). However, no statistically significant difference was found in CMT between the posttreatment third and the sixth months (Paired $\mathrm{t}$ test; $\mathrm{p}=0.063)$. Changes in mean RNFLT and CMT were shown in Table 2.

\section{DISCUSSION}

It is well-proven that PRP prevents severe visual loss in patients with proliferative DR, however laser treatment is fraught with several complications mostly stemmed from retinal inflammation triggered by laser burns itself [14]. Hiroshiba et al. [15] reported that hemodynamics of retinal capillary system was disrupted due to chemical mediators secreted from the photocoagulated tissue. Irreversible injury was detected in perivascular axons of Rhesus monkeys after direct photocoagulation over the arteries and venules, while tissue response may vary from minimal destruction of the outer retina to full-thickness damage involving RNFL with similar laser parameters in areas free of major vessels [16].

OCT is the most sensitive and quantitative test for the evaluation of retinal and especially macular edema. Spectraldomain OCT devices help to make more precise assesment of RNFLT with respect of retinal quadrants, as they provide high defination images of retinal layers [17]. Maia et al. [18] performed RNFLT evaluation with time-domain OCT at the first week as well as the first, third and sixth months after PRP. They demonstrated that any reduction of RNFLT had not been observed in the short and medium term of photocoagulation, however a significant increase in temporal RNFLT, especially at 3 and 4 o'clock sectors, was observed up to six months postPRP, without any reduction in RNFLT after 6 months. Kim and Cho [19] found that there was no statistically significant difference in time-domain based RNFLT scores when the mean RNFLT of 118 patients with severe nonproliferative DR at the sixth month of postlaser was compared with mean RNFLT scores of 164 diabetic patients without retinopathy. Although statistically significant decrease in RNFLT was found at 6 months after PRP treatment comparing with baseline scores in patients with DR.

In a time-domain OCT based retrospective chart review conducted by Kim et al. [6], statistically significant decrease in the average RNFLT at 2 years after PRP treatment was reported, whereas the mean RNFLT score slightly increased during the initial 3 months after photocoagulation. Authors reported statistically significant reduction in RNFLT within the superior and inferior quadrants at 2 years post-PRP as well as they found a borderline significance in the decrease of nasal quadrant RNFLT at 2 years after photocoagulation; however any reduction in RNFLT within the temporal quadrant was not observed up to 36 months post-PRP. Kim et al. [6] reported that RNFLT underwent early thickening and thereafter progressive thinning during the 2 years after photocoagulation. In our study, as distinct from the remarkable RNFLT increase in the other peripapillary quadrants, no statistically significant increase was found in temporal RNFLT at the third month of posttreatment followup. However spectral-domain OCT showed us a marked RNFLT decrease in all of the four peripapillary quadrants at the sixth post-PRP month. Muqit et al. [9] prospectively evaluated the RNFLT alterations on 10 eyes with proliferative DR after PRP treatment performed with $514 \mathrm{~nm}$ green laser. In order to measure RNFLT in the peripapillary zone, the Stratus OCT3 system was used in their study. Authors reported statistically significant RNFLT increase $(+8 \mu \mathrm{m} ; \mathrm{p}<0.05)$ at 10 weeks after photocoagulation, however significant reduction in RNFLT $(-4 \mu \mathrm{m} ; \mathrm{p}<0.05)$ was measured after the sixth month of posttreatment followup. They attributed the early thickening of RNFL following the PRP to inner retinal axon damage by thermal diffusion around the 100-ms burn, disruption of the mid-flow axonal flow and axonal edema [9].

In accordance with the previous studies, we observed a significant RNFLT increase at the third month of postlaser follow-up, however significant RNFLT reduction was also detected in each retinal quadrants at six months post-PRP in our study population. In the present study, statistically significant increase in RNFLT was noted at the third post-laser month compared to its baseline values within each quadrants except temporal retina. Nevertheless we observed a significant reduction in RNFLT within each retinal quadrants at the sixth post-laser month compared to its baseline values. Acute edema related increase in retinal thickness develops soon after the photocoagulation, and it may protect ganglion cells against the destructive effects of laser application, so retinal injury worsens in case of repetitious laser treatments with longer intervals [20]. Other risks of photocoagulation are the fusion and expansion of laser burns in late phases. Laser burns may expand even until the fourth year of posttreatment follow-up, and the annual rate for laser burn expansion was found to be as high as $16.5 \%$. Therefore progressive destruction in RNFL and visual field loss can be seen after PRP treatment [21]. Hence, choosing the sufficient parameters for optimum efficacy and safety is mandatory. Since lower energy is delivered with the application of $20 \mathrm{~ms}$ PASCAL laser comparing to traditional PRP treatment, Muqit et al. [22] reported the prevention of excessive neuroretinal tissue loss with PASCAL photocoagulation in their fourier-domain OCT based prospective study. In PASCAL single-session PRP group, no significant change in total RNFLT from its baseline values was found ( 4 weeks: $+7.2 \mu \mathrm{m}, \mathrm{p}=0.78$; 12 weeks: $-1.8 \mu \mathrm{m}, \mathrm{p}=0.95)$; however in multiple-session PRP group, total RNFLT alterations were significant at both the first and third months of posttreatment follow-up when compared with the baseline scores ( 4 weeks: $+31 \pm 54 \mu \mathrm{m}, \mathrm{p}=0.029 ; 12$ weeks: $-35 \pm 63 \mu \mathrm{m}, \mathrm{p}=0.034)$. In another study conducted by Muqit et al. [23], significant reductions in CMT were found on fourier-domain OCT after PASCAL-targeted retinal photocoagulation, as well as after reduced-fluence PRP when compared to CMT changes after standart-intensity PRP. Nevertheless the authors did not report any significant changes in BCVA and RNFLT among three study groups at 12 weeks after laser treatment. In our study population, decrease in CMT was noted in both the third and sixth months of posttreatment follow-up with the mean of $-5.8 \mu \mathrm{m}$ and $-8.2 \mu \mathrm{m}$, respectively. There is a conventional concern on PRP induced macular edema. However laser treatment may also decrease VEGF dry 
by reducing the area of ischemia and has a positive effect on CMT particularly in patients without significant macular edema at the start of PRP.

Although visual acuity of patients who underwent PRP had a tendency for improvement, no statistical significant difference was found between final and initial BCVA scores in our treatment group. However visual acuity was decreased in 4 eyes (6.9\%), visual stabilization or improvement was achieved in 54 eyes $(93.1 \%)$ at 6 months post-PRP. Reports of ETDRS revealed diminished vision in $26 \%$ of patients in whom PRP treatment was not given, whereas only $11 \%$ of the patients who underwent photocoagulation experienced with a decrease in their visual acuity [24]. Two or more lines of decrease in vision due to PRP induced macular edema was reported in $8 \%$ of their patients by McDonald and Schatz [25]. Comparing with the literature, our treatment group had better visual outcomes which might be due to our patient selection criteria, as cases who required combined intravitreal injections for severe macular edema were deliberately excluded.

\section{CONCLUSION}

Increase in RNFLT at the third month of follow-up may be related to laser induced intraretinal inflammation which triggers increased capillary permeability and ensuing axonal edema due to the cytokine release. Significant RNLFT decrease at the sixth month of follow-up can be attributed to axonal loss secondary to direct or indirect effects of PRP treatment. Spectral-domain OCT is a very valuable tool to document the RNFLT changes following the panretinal photocoaculation.

\section{CONFLICT OF INTEREST}

The authors confirm that this article content has no conflict of interest.

\section{ACKNOWLEDGEMENTS}

Declared none.

\section{REFERENCES}

Kein R, Klein BE, Moss SE, Davis MD, DeMets DL. The Wisconsin epidemiologic study of diabetic retinopathy. IV. Diabetic macular edema. Ophthalmology 1984; 91(12): 1464-74.

[2] Photocoagulation treatment of proliferative diabetic retinopathy. Clinical application of Diabetic Retinopathy Study (DRS) findings, DRS Report Number 8. The Diabetic Retinopathy Study Research Group. Ophthalmology 1981; 88(7): 583-600.

[3] Photocoagulation treatment of proliferative diabetic retinopathy: relationship of adverse treatment effects to retinopathy severity. Diabetic retinopathy study report no. 5. Dev Ophthalmol 1981;2: 24861.

[4] Steffanson E. Oxygen and diabetic eye disease. Graefes Arch Clin Exp Ophthalmol 1990; 228: 120-3.

[5] Schatz H, Madeira D, McDonald HR, Johnson RN. Progressive enlargement of laser scars following grid laser photocoagulation for diffuse diabetic macular edema. Arch Ophthalmol 1991; 109: 1549-51.

[6] Kim J, Woo SJ, Ahn J, Park KH, Chung H, Park KH. Long-term temporal changes of peripapillary retinal nerve fiber layer thickness before and after panretinal photocoagulation in severe diabetic retinopathy. Retina 2012; 32: 2052-60.

[7] Shimura M, Yasuda K, Nakazawa T, et al. Panretinal photocoagulation induces pro-inflammatory cytokines and macular thickening in highrisk proliferative diabetic retinopathy. Graefes Arch Clin Exp Ophthalmol 2009; 247: 1617-24.

[8] Lim MC, Tanimoto SA, Furlani BA, et al. Effect of diabetic retinopathy and panretinal photocoagulation on retinal nerve fiber layer and optic nerve appearance. Arch Ophthalmol 2009; 127(7): 857-62.

[9] Muqit MM, Wakely L, Stanga PE, Henson DB, Ghanchi FD. Effects of conventional argon panretinal laser photocoagulation on retinal nerve fibre layer and driving visual fields in diabetic retinopathy. Eye 2010; 24: $1136-42$.

[10] Lee SB, Kwag JY, Lee HJ, Jo YJ, Kim JY. The longitudinal changes of retinal nerve fiber layer thickness after panretinal photocoagulation in diabetic retinopathy patients. Retina 2013; 33(1): 188-93.

[11] Langenegger SJ, Funk J, Töteberg-Harms M. Reproducibility of retinal nerve fiber layer thickness measurements using the eye tracker and the retest function of Spectralis SD-OCT in glaucomatous and healthy control eyes. Invest Ophthalmol Vis Sci 2011; 52: 3338-44.

[12] Wu H, de Boer JF, Chen TC. Reproducibility of retinal nerve fiber layer thickness measurements using spectral domain optical coherence tomography. J Glaucoma 2010; 19: 226-7.

[13] Lavinsky D, Cardillo JA, Mandel Y, et al. Restoration of retinal morphology and residual scarring after photocoagulation. Acta Ophthalmol 2013; 91(4): e315-23

[14] Mendivil A, Cuartero V, Mendivil MP. Ocular blood flow velocities in patients with proliferative diabetic retinopathy before and after scatter photocoagulation: a prospective study. Eur J Ophthalmol 1995; 5: 25964.

[15] Hiroshiba N, Ogura $\mathrm{Y}$, Nishiwaki H, Miyamoto K, Honda $\mathrm{Y}$, Alterations of retinal microcirculation in response to scatter photocoagulation. Invest Ophthalmol Vis Sci 1998; 39(5): 769-76.

[16] Apple DJ, Wyhinny GJ, Goldenberg MF, Polley EH, Bizzell JW. Experimental argon laser photocoagulation. I. Effects on retinal nerve fiber layer. Arch Ophthalmol 1976; 94(1): 137-44.

[17] Querques G, Forte R, Berboucha E, et al. Spectral-domain versus time domain optical coherence tomography before and after ranibizumab for age-related macular degeneration. Ophthalmic Res 2011; 46(3): 152-9.

[18] Maia OO Jr, Vessani RM, Takahashi WY, Susanna R Jr. Examination of the retinal nerve fiber layer in diabetic retinopathy treated by argon laser panphotocoagulation. Arq Bras Oftalmol 2008; 71(2): 187-90.

[19] Kim HY, Cho HK. Peripapillary retinal nerve fiber layer thickness change after panretinal photocoagulation in patients with diabetic retinopathy. Korean J Ophthalmol 2009; 23: 23-6.

[20] Tonello M, Costa RA, Almeida FP, Barbosa JC, Scott IU, Jorge R. Panretinal photocoagulation versus PRP plus intravitreal bevacizumab for high-risk proliferative diabetic retinopathy (IBeHi study). Acta Ophthalmol 2008; 86(4): 385-9.

[21] Maeshima K, Utsugi-Sutah N, Otani T. Progressive enlargement of scattered photocoagulation scars in diabetic retinopathy. Retina 2004; 24: 507-11.

[22] Muqit MM, Marcellino GR, Henson DB, Fenerty CH, Stanga PE. Randomized clinical trial to evaluate the effects of Pascal panretinal photocoagulation on macular nerve fiber layer: Manchester Pascal Study report 3. Retina 2011; 31(8): 1699-707.

[23] Muqit MM, Young LB, McKenzie R, et al. Pilot randomised clinical trial of Pascal TargETEd Retinal versus variable fluence PANretinal 20 ms laser in diabetic retinopathy: PETER PAN study. Br J Ophthalmol 2013; 97(2): 220-7.

[24] Early photocoagulation for diabetic retinopathy. ETDRS report number 9. Early treatment diabetic retinopathy study research group. Ophthalmology 1991; 98(5 Suppl): 766-85.

[25] McDonald HR, Schatz H. Macular edema following panretinal photocoagulation. Retina 1985; 5(1): 5-10. 